\section{MS16 P01}

Flexible supramolecular ring-like host for various tetrahedral anion guests Dubravka MatkovićČalogović, Krunoslav Užarević, Ivica Đilović, Marina Cindrić, Laboratory of General and Inorganic Chemistry, Chemistry Department, Faculty of Science, University of Zagreb, Zagreb, Croatia. E-mail: dubravka@chem.pmf.hr

Keywords: supramolecular receptor, anion binding, hydrogen bond

Anion binding by synthetic receptors is a rapidly growing area of interest in supramolecular chemistry. Such research enables better understanding of the role of anion binding in biological systems ${ }^{1}$.

The design of anion receptors is challenging ${ }^{2}$. Anions can be $\mathrm{pH}$ sensitive, complex anions can have different geometries. Therefore, anion receptors must function in active $\mathrm{pH}$ range of anions. Also, it is optimal for the receptors to have flexible geometry and so become complementary to various anion guests.

In our work, we prepared a supramolecular host for two tetrahedral anions: $\mathrm{SO}_{4}{ }^{2-}$ and $\mathrm{ClO}_{4}^{-}$. The crystals are unstable upon exposure to air so the single crystal diffraction data were collected from the crystal mounted in a loop in a mixture of methanol and glycerol, and cooled in nitrogen vapour stream at $100 \mathrm{~K}$. The structures revealed that the ring-like host is formed by three molecules which have flexible parts. When sulphate anion is the guest it occupies the center of the ring since the hydrophilic parts of the molecules are at the inside of the formed host ring (A). The disordered sulphate anion forms 12 hydrogen bonds with the host molecules. On contrary, when perchlorate anion is the guest it occupies voids between the rings formed by three molecules which are now folded in such a way that the methyl groups point inward leaving no space within the ring for the guest (B).

A

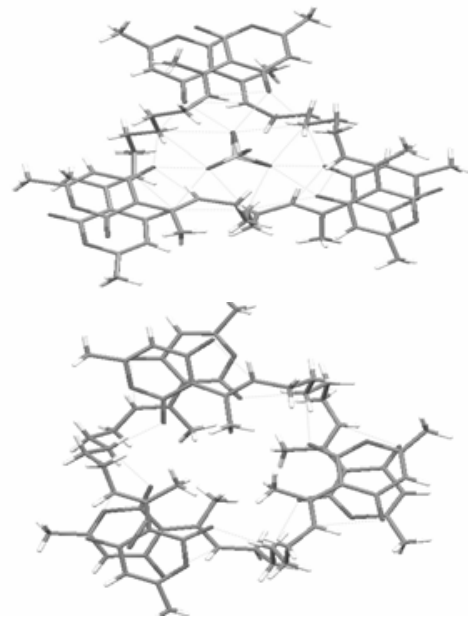

[1] Bowman-James K. et al., Angew.Chem.Int.Ed. 2006, 45, 7882. [2] Beer P.D., Gale P.A., Angew.Chem.Int.Ed. 2001, 40, 487.

\section{MS16 P02}

Structural aspects of derivative chiral separation Petra Bombicz $^{\mathrm{a}}$, Laura Bereczki ${ }^{\mathrm{b}}$, Katalin Marthic ${ }^{\mathrm{c}}$, György Pokol $^{\mathrm{b}}$, Elemér Fogassyd, 'anstitute of Structural Chemistry, Chemical Research Center, Hungarian Academy of Sciences, Hungary. ${ }^{b}$ Department of Inorganic and Analytical Chemistry, Budapest University of Technology and Economics, Hungary. ${ }^{c}$ Research Group of Technical Analytical Chemistry, Hungarian Academy of
Sciences - Budapest University of Technology and Economics, Hungary. ${ }^{d}$ Department of Organic Chemistry and Technology, Budapest University of Technology and Economics, Hungary. E-mail: bombicz@chemres.hu

\section{Keywords: chiral separation, intermolecular interactions, columnar structures}

Chiral separation depends on complex supramolecular recognition processes and the outcome is influenced by coincidence of numerous fine tuned intermolecular interactions, therefore the expected result of a given procedure is hardly predictable. The resolving agent can be obtained by derivatizing the pure enantiomers to get a derivative with opposite acid-base character. Use of a derivative resolving agent is promising, since the related molecular structures of the racemate and the resolving agent provide compatibility of the molecular shape and the electron distribution of the molecules that makes an efficient molecular recognition possible.

Only few X-ray crystal structures of pairs of diastereomeric salts are available and even less is the number of the structures of homologues of diastereomers. Single crystal X-ray diffraction structures of $(R)$ - and $(S)$ 1-(1-naphthyl)-ethylammonium $\quad(R)-N$-[1-(1naphthyl)ethyl]oxalamate salts [1] are compared to structures of $(R)$ - and $(S)$-1-phenyl-ethylammonium $(R)-N$ (1-phenylethyl)oxalamates [2]. The size of the aromatic substituent is a factor with secondary importance, although its shape determines the possibility of the chiral separation. The most important are the changes that affect the polar substituents of the molecules.

$(S)$-(-)-1-phenylethylammonium $(S)-(+)-N$-formylphenylalaninate salt is an example for the appearance of kinetic control in optical resolutions by diastereomeric salt formation [3]. The arrangement of the infinite molecular columns is strongly dependent on weaker secondary interactions determining the rate of crystal growth consequently the resolution efficiency.

All crystal structures are closely similar in the respect that they are built up of inner hydrophilic, outer hydrophobic homochiral columns running along the screw axis. The infinite inner hydrogen bond system is hardly influenced by the aromatic substituent. The outer hydrophobic "coating" provides directed steric arrangements. The arrangement of the columns is determined by weak intermolecular interactions.

(The work was supported by Hungarian Research Fund (OTKA) grants T042642, T042725 and F037814.)

[1] Bereczki, L., Bombicz, P., Bálint, J., Egri, G., Pokol, G., Fogassy, E., Marthi, K,_Chirality In preparation 2007.

[2] Bálint J, Egri G, Czugler M, Schindler J, Kiss V, Juvancz Z, Fogassy E., Tetrahedron-Asymmetry 2001, 12, 1511.

[3] Bereczki, L., Pálovics, E., Bombicz, P., Pokol, G., Fogassy, E., Marthi, K.,_Tetrahedron Asymmetry, 2007, 18, 260. 